\title{
Logic and intelligent interaction
}

\author{
Thomas Ågotnes · Johan van Benthem • \\ Eric Pacuit
}

Received: 8 April 2009 / Accepted: 13 April 2009 / Published online: 7 May 2009

(C) The Author(s) 2009. This article is published with open access at Springerlink.com

You can think about planet Earth and its natural place for centuries, but to see how it really moves, you need to know the solar system, and all the bodies that interact with it. The basic laws of gravity are about 'social' push and pull. And what is true of physical bodies, is even more true of our human world, where bodies also have minds. You can think about human beings as isolated information processors as much as you want, turning them into idealized mathematicians serving a life sentence in Platonic Heaven, but if you want to understand the dynamics that gives human actions sense, you need to understand our information about and responses to others. And indeed, there is a fast-growing interest in dynamic logics that deal with 'intelligent interaction' (the happy title of a current EC strategic research program ${ }^{1}$ ) in communities of rational, or perhaps not so rational agents. There is also a fast-growing jungle of formal systems, sometimes with a 'religious' following as so often is the case in early stages of a field.

The Workshop that led to the present volume was held at the 2008 European Summer School of Logic, Language and Information (ESSLLI) in Hamburg, August 11-15. ${ }^{2}$

1 See http://www.esf.org/activities/eurocores/programmes/logiccc.html for more information.

2 See http://ai.stanford.edu/ epacuit/LaII for a full program and list of contributed talks.

This is the editorial for the KRA special issue on LOII.

T. Ågotnes $(\varangle)$

Department of Information Science and Media Studies, University of Bergen, Bergen, Norway e-mail: thomas.agotnes@infomedia.uib.no

J. van Benthem

Institute for Logic, Language and Computation, University of Amsterdam, Amsterdam, Netherlands e-mail: johan@science.uva.nl

E. Pacuit

Department of Philosophy, Stanford University, California, USA

e-mail: epacuit@stanford.edu 
It was dedicated to taking the pulse of the field by looking at recent convergences between logical frameworks for rational agency, and more generally, trying to foster a common sense of what is going on. Incidentally, many of these frameworks have a computational background and this makes sense, since it might plausibly be claimed that the phenomenon of 'agency' is to modern logic what 'computation' was to the earlier foundational phase of the discipline. In addition to narrower methodological aims, the talks gave $80+$ workshop participants a glimpse of a very lively set of research themes cooking today, often at the interface of logic, computer science, philosophy, and game theory. Judging from positive responses at the scene, an encounter of bodies had become a meeting of the minds.

The papers in this issue were selected from presentations at the workshop, and then survived a standard refereeing process.

Keep 'hoping' for rationality: a solution to the backward induction paradox by Alexandru Baltag, Sonja Smets, and Jonathan Zvesper uses dynamic-epistemic logics of information flow to analyze game solution procedures, finding new twists to Aumann's characterization of backward induction. Successive learning acts in doxastic plausibility models for extensive games lead to "stable belief" in dynamic rationality during play, which eventually results in common knowledge of stable belief in rationality.

Reasoning about cooperation, action and preferences by Lena Kurzen analyzes rich logics of cooperative agency that go beyond current 'coalition logics' by including the crucial phenomenon of individual and group preferences providing the deeper dynamics driving agents' decisions and the resulting interaction. A new logical system is developed for reasoning about explicitly preference-based coalitional power, which is then axiomatized, while its satisfiability problem is shown to be NEXPTIMEcomplete.

Determining the environment: a modal logic for closed interaction by Jan Broersen, Rosja Mastop, John-Jules Meyer and Paolo Turrini provides a language to reason about closed-world interactions. That is, all those situations in which the outcomes of an interaction can be determined by the agents themselves and in which the environment cannot interfere with their choices. They identify all such interactions and axiomatize the underlying logic. The formal tools are used to reason about games and their regulation.

Bridging learning theory and dynamic epistemic logic by Nina Gierasimczuk discusses ways of modelling inductive inference from formal learning theory, an influential account of long-term informational processes, in terms of dynamic-epistemic, or dynamic-doxastic logic. Epistemological notions involved in identification in the limit are matched with logical statements, and a precise match is made between 'learning by erasing' and iterated belief revision by dynamic-doxastic update rules.

Intentions and interactive transformations of decision problems by Olivier Roy enriches standard models of decisions and games with notions of 'intention'. There are two main ways of transforming decision problems on the basis of previous intentions: ruling out incompatible options and imposing a standard of relevance. In the multiagent setting, these can leave agents with no option compatible with what they intend, but coherence is restored when agents consider the intentions of others. 
Inference and update by Fernando Velazquez Quesada high-lights two fundamental logical processes, often intertwined in planning and problem solving: inference and update. Inference is an internal process of elucidation, while update is produced by external observation or communication. Their integration is a long-standing problem in the philosophy of logic. Both processes can be described in one dynamic logic, merging dynamic-epistemic logic with logics of awareness-raising inference steps.

Introspective forgetting by Hans van Ditmarsch, Andreas Herzig, Jerome Lang and Pierre Marquis model the forgetting of propositional variables in a modal logical context where agents become ignorant and are aware of each others' or their own resulting ignorance. The resulting logic is sound and complete. It can be compared to variable-forgetting as abstraction from information, wherein agents become unaware of certain variables. By employing elementary results for bisimulation, it follows that beliefs not involving the forgotten atom(s) remain true.

Dynamic epistemic logic with branching temporal structures by Tomohiro Hoshi and Audrey Yap extends recent connections between epistemic temporal logic and dynamic epistemic logic of events iterated along protocols. They add a past-time operator to the system, which allows for stating more realistic preconditions of events, as well as more sophisticated specifications of the relevant informational process. They then axiomatize the resulting extension of standard DEL-generated epistemic temporal logics, whose expressive power gets closer to, amongst others, that of learning theory.

Extending probabilistic dynamic epistemic logic by Joshua Sack extends earlier probabilistic dynamic epistemic logics in two directions. The first extension adds a past-time operator to include more process structure, the second involves a use of nontrivial $\sigma$-algebras to deal with more scenarios than the usual finite probability spaces. The resulting merge of ideas from dynamic logics and probability theory can handle more significant scenarios from the literature than the toy systems available so far.

Verifying time, memory and communication bounds in systems of reasoning agents by Natasha Alechina, Brian Logan, Hoang Nga Nguyen and Abdur Rakib develops a logical framework for verifying systems composed of agents that may have differing knowledge and inferential capabilities, and where the resources (time, memory and communication bandwidth) are bounded. They present a novel epistemic temporal logic that describes a set of reasoning agents with bounds on time, memory and the number of messages they can exchange. They show, among other things, how to axiomatize a system of agents which reason using resolution and prove that the resulting logic is sound and complete.

We trust that these papers convey the spirit of the workshop, and we hope that readers of KRA will find them as congenial and inspiring as we did.

Open Access This article is distributed under the terms of the Creative Commons Attribution Noncommercial License which permits any noncommercial use, distribution, and reproduction in any medium, provided the original author(s) and source are credited. 